\title{
ON GREEN'S FUNCTION OF AN $n$-POINT BOUNDARY VALUE PROBLEM
}

BY

\author{
K. M. DAS AND A. S. VATSALA
}

ABSTRACT. The Green's function $g_{n}(x, s)$ for an $n$-point boundary value problem, $y^{(n)}(x)=0, y\left(a_{1}\right)=y\left(a_{2}\right)=\cdots=y\left(a_{n}\right)=0$ is explicitly given. As a tool for discussing $\operatorname{sgn} g_{n}(x, s)$ on the square $\left[a_{1}, a_{n}\right] \times\left[a_{1}, a_{n}\right]$, some results about polynomials with coefficients as symmetric functions of $a^{\prime}$ 's are obtained. It is shown that

$$
\int_{a_{1}}^{a_{n}}\left|g_{n}(x, s)\right| d s
$$

is a suitable polynomial in $x$. Applications to $n$-point boundary value problems and lower bounds for $a_{m}(m \geq n)$ are included.

1. Introduction. Beesack [1] considered the boundary value problem

$$
\begin{aligned}
y^{(n)}(x) & =0, \\
y\left(a_{i}\right) & =y^{\prime}\left(a_{i}\right)=\cdots=y^{\left(k_{i}\right)}\left(a_{i}\right)=0 \quad(1 \leq i \leq r),
\end{aligned}
$$

where $a_{1}<a_{2}<\ldots<a_{r}, 0 \leq k_{i}, k_{1}+k_{2}+\cdots+k_{r}=n-r$. For the Green's function $g_{n}(x, s)$ he proved that

$$
\left|g_{n}(x, s)\right| \leq \frac{\Pi_{i=1}^{r}\left|x-a_{i}\right|^{k_{i}+1}}{(n-1) !\left(a_{r}-a_{1}\right)} .
$$

In [2] Nehari gave a short proof of the same when $r=n$. Since in relation to multipoint boundary value problem as in [1], $\int_{a_{1}}^{a_{r}}\left|g_{n}(x, s)\right| d s$ appears (see 3.5 there), the natural question is to consider alternately this function.

In this paper, we consider (1.1) when $r=n$ first. In $\$ 2$, we give the Green's function $g_{n}(x, s)$ explicitly and alternately exhibit it in a form which yields conclusions as to the sign of $g_{n}(x, s)$. In $\$ 3$, the results about $\operatorname{sgn} g_{n}(x, s)$ and the identity

Received by the editors March 17, 1972 and, in revised form, December 11, 1972. AMS (MOS) subject classifications (1970). Primary 34B10; Secondary 12D10, $26 A 82$.

Key words and phrases. Green's function, multipoint boundary value problem, zeros of solutions. 


$$
\int_{a_{1}}^{a_{n}}\left|g_{n}(x, s)\right| d s=\frac{1}{n !}\left(x-a_{1}\right)\left(a_{n}-x\right) \prod_{i=2}^{n-1}\left|x-a_{i}\right|
$$

are obtained. (There are a few auxiliary results given as lemmas which may be of some independent interest!) Applications to $n$-point boundary value problems and lower bounds for the $m$ th zero of solutions form the contents of $\S 4$.

2. The Green's function. Throughout, $n$ denotes a fixed natural number greater than 2 . Let $k$ be a natural number such that $2<k \leq n$. Consider the boundary value problem

$$
\begin{aligned}
y^{(k)}(x) & =0, \\
y\left(a_{1}\right) & =y\left(a_{2}\right)=\cdots=y\left(a_{k-1}\right)=y\left(a_{n}\right)=0,
\end{aligned}
$$

where $a_{1}<a_{2}<\ldots<a_{k-1}<a_{n}$.

Theorem 2.1. The Green's function $g_{k}(x, s)$ for (2.1) is given by

$$
\begin{aligned}
&(k-1) ! g_{k}(x, s) \\
&=\left(\prod_{i=1}^{k-1} \frac{x-a_{i}}{a_{n}-a_{i}}\right)\left(a_{n}-s\right)^{k-1}, \quad x \leq s, a_{k-1} \leq s ; \\
&=\left(\prod_{i=1}^{k-1} \frac{x-a_{i}}{a_{n}-a_{i}}\right)\left(a_{n}-s\right)^{k-1} \\
&+\sum_{j=2}^{r+1}(-1)^{j}\left(\prod_{\substack{i=1 \\
(i \neq k-j+1)}}^{k-1} \frac{x-a_{i}}{\left|a_{k-j+1}-a_{i}\right|}\right) \frac{a_{n}-x}{a_{n}-a_{k-j+1}}\left(a_{k-j+1}-s\right)^{k-1} \\
&\left(\equiv \varepsilon_{k}^{r}(x, s)\right), \quad x \leq s, a_{k-r-1} \leq s \leq a_{k-r}(r=1, \ldots, k-3) ;
\end{aligned}
$$

$$
\begin{aligned}
& =\left(\prod_{i=2}^{k-1} \frac{x-a_{i}}{a_{i}-a_{1}}\right) \frac{a_{n}-x}{a_{n}-a_{1}}\left(s-a_{1}\right)^{k-1}+(-1)^{k-1}(s-x)^{k-1}, \quad x \leq s, s \leq a_{2} ; \\
& =\left(\prod_{i=1}^{k-1} \frac{x-a_{i}}{a_{n}-a_{i}}\right)\left(a_{n}-s\right)^{k-1}-(x-s)^{k-1}, \quad a_{k-1} \leq s \leq x ; \\
& =g_{k}^{r}(x, s)-(x-s)^{k-1}, \quad s \leq x, a_{k-r-1} \leq s \leq a_{k-r}(r=1, \ldots, k-3) ; \\
& =\left(\prod_{i=2}^{k-1} \frac{x-a_{i}}{a_{i}-a_{1}}\right) \frac{a_{n}-x}{a_{n}-a_{1}}\left(s-a_{1}\right)^{k-1}, \quad s \leq x, s \leq a_{2} ;
\end{aligned}
$$

where a product for empty set of indices is interpreted as 1 . 
Remark. Here, as well as in the following, $r$ ranging over a vacuous set of indices means the collapse of regions of the form $x \leq s \quad(x \geq s), a_{k-r-1} \leq s \leq$ $a_{k-r}$.

Proof. Starting with the Green's function for $y^{\prime \prime}(x)=0, y\left(a_{1}\right)=y\left(a_{2}\right)=0$, namely

$$
g_{2}(x, s)= \begin{cases}\left(x-a_{1}\right)\left(a_{n}-s\right) /\left(a_{n}-a_{1}\right), & x \leq s \\ \left(a_{n}-x\right)\left(s-a_{1}\right) /\left(a_{n}-a_{1}\right), & s \leq x\end{cases}
$$

and the relation in [1],

$$
g_{m+1}(x, s)=\frac{1}{m}\left\{(x-s) g_{m}(x, s)-\left(a_{m}-s\right) g_{m}\left(a_{m}, s\right)\left(\prod_{i=1}^{m-1} \frac{x-a_{i}}{a_{m}-a_{i}}\right) \frac{a_{n}-x}{a_{n}-a_{m}}\right\},
$$

it is easily checked that

$$
2 ! g_{3}(x, s)= \begin{cases}\frac{\left(x-a_{1}\right)\left(x-a_{2}\right)}{\left(a_{n}-a_{1}\right)\left(a_{n}-a_{2}\right)}\left(a_{n}-s\right)^{2}, \quad x \leq s, a_{2} \leq s \\ \frac{\left(x-a_{2}\right)\left(a_{n}-x\right)}{\left(a_{2}-a_{1}\right)\left(a_{n}-a_{1}\right)}\left(s-a_{1}\right)^{2}+(s-x)^{2}, \quad x \leq s, s \leq a_{2} \\ \frac{\left(x-a_{1}\right)\left(x-a_{2}\right)}{\left(a_{n}-a_{1}\right)\left(a_{n}-a_{2}\right)}\left(a_{n}-s\right)^{2}-(x-s)^{2}, \quad s \leq x, a_{2} \leq s \\ \frac{\left(x-a_{2}\right)\left(a_{n}-x\right)}{\left(a_{2}-a_{1}\right)\left(a_{n}-a_{1}\right)}\left(s-a_{1}\right)^{2}, \quad s \leq x, \quad s \leq a_{2} .\end{cases}
$$

Thus (2.2) is valid for $k=3$. Again, assuming that (2.2) holds when $k=m$, if $x \leq s, a_{m} \leq s$, then (2.3) gives

$$
\begin{aligned}
m ! g_{m+1}(x, s) & =\left(\prod_{i=1}^{m-1} \frac{x-a_{i}}{a_{n}-a_{i}}\right)\left\{(x-s)-\left(a_{m}-s\right) \frac{a_{n}-x}{a_{n}-a_{m}}\right\}\left(a_{n}-s\right)^{m-1} \\
& =\left(\prod_{i=1}^{m} \frac{x-a_{i}}{a_{n}-a_{i}}\right)\left(a_{n}-s\right)^{m}
\end{aligned}
$$

Similarly, if $x \leq s, a_{m-r} \leq s \leq a_{m-r+1}$, where $r=2, \cdots,(m+1)-3,(2.3)$ yields 


$$
\begin{aligned}
& m ! g_{m+1}(x, s) \\
& =\left((x-s)-\left(a_{m}-s\right) \frac{a_{n}-x}{a_{n}-a_{m}}\right)\left(\prod_{i=1}^{m-1} \frac{x-a_{i}}{a_{n}-a_{i}}\right)\left(a_{n}-s\right)^{m-1}+\left(\prod_{i=1}^{m-1} \frac{x-a_{i}}{a_{m}-a_{i}}\right) \frac{a_{n}-x}{a_{n}-a_{m}}\left(a_{m}-s\right)^{m} \\
& +\sum_{j=2}^{r}(-1)^{j}\left(\prod_{\substack{i=1 \\
i \neq m-j+1)}}^{m-1} \frac{x-a_{i}}{\left|a_{m-j+1}-a_{i}\right|}\right) \frac{a_{n}-x}{a_{n}-a_{m-j+1}} \\
& \cdot\left(a_{m-j+1}-s\right)^{m-1}\left((x-s)-\left(a_{m}-s\right) \frac{x-a_{m-j+1}}{a_{m}-a_{m-j+1}}\right) \\
& =-\left(\prod_{i=1}^{m} \frac{x-a_{i}}{a_{n}-a_{i}}\right)\left(a_{n}-s\right)^{m}+\sum_{j=1}^{r}(-1)^{j+1}\left(\prod_{\substack{i=1 \\
i \leqslant m-j+1}}^{m} \frac{x-a_{i}}{\left|a_{m-j+1}-a_{i}\right|}\right) \frac{a_{n}-x}{a_{n}-a_{m-j+1}}\left(a_{m-j+1}-s\right)^{m} .
\end{aligned}
$$

A similar computation using the first expression of (2.2) with $k=m$ gives the result for $x \leq s, a_{m-1} \leq s \leq a_{m}$. Moreover, if $x \leq s \leq a_{2}$, then

$$
\begin{aligned}
& m !_{m+1}(x, s) \\
& =(-1)^{m}(s-x)^{m}+\left(\prod_{i=2}^{m-1} \frac{x-a_{i}}{a_{i}-a_{1}}\right) \frac{a_{n}-x}{a_{n}-a_{1}}\left(s-a_{1}\right)^{m-1}\left((x-s)-\left(a_{m}-s\right) \frac{x-a_{1}}{a_{m}-a_{1}}\right) \\
& =\left(\prod_{i=2}^{m} \frac{x-a_{i}}{a_{i}-a_{1}}\right) \frac{a_{n}-x}{a_{n}-a_{1}}\left(s-a_{1}\right)^{m}+(-1)^{m}(s-x)^{m} .
\end{aligned}
$$

This completes the induction argument for the triangle $x \leq s$. On the same lines the region $a_{1} \leq s \leq x \leq a_{n}$ can be handled. Hence the conclusion.

Corollary 2.2. Alternatively, if $s \leq x$ and $a_{k-r-1} \leq s \leq a_{k-r}$ where $r=$ $1, \ldots, k-3$, we bave

$$
\begin{aligned}
(k-1) ! g_{k}(x, s)=\left(a_{n}-x\right) & \sum_{l=0}^{k-r-2}(x-s)^{l}\left(\prod_{i=l+2}^{k-1}\left(x-a_{i}\right)\right)\left(s-a_{l+1}\right) \\
& \left\{\frac{\left(a_{n}-s\right)^{k-l-2}}{\prod_{i=l+1}^{k-1}\left(a_{n}-a_{i}\right)}+\sum_{j=2}^{r+1}(-1)^{j+1} \frac{\left(a_{k-j+1}-s\right)^{k-l-2}}{\Pi_{i=l+1(i \neq k-j+1)}^{k-1}\left|a_{k-j+1}-a_{i}\right|} \frac{1}{a_{n}-a_{k-j+1}}\right\} .
\end{aligned}
$$

Proof. First observe that $\left(x-a_{i}\right)\left(a_{m}-s\right)=\left(a_{m}-a_{i}\right)(x-s)+\left(s-a_{i}\right)\left(a_{m}-x\right)$, $i \neq m$. Applying this first with $i=1$ and $m=n$ or $k-j+1$-note that $m$ is always different from 1-, we get 


$$
\begin{aligned}
g_{k}^{r}(x, s)-(x-s)^{k-1}=\left(a_{n}-x\right) & {\left[\left(\prod_{i=2}^{k-1}\left(x-a_{i}\right)\right)\left(s-a_{1}\right)\right.} \\
& \left.\cdot\left\{\frac{\left(a_{n}-s\right)^{k-2}}{\prod_{i=1}^{k-1}\left(a_{n}-a_{i}\right)}+\sum_{j=2}^{r+1}(-1)^{j+1} \frac{\left(a_{k-j+1}-s\right)^{k-2}}{\prod_{i=1(i \neq k-j+1}^{k-1}\left|a_{k-j+1}-a_{i}\right| a_{n}-a_{k-j+1}}\right)\right] \\
+(x-s) & {\left.\left[\prod_{i=2}^{k-1} \frac{1}{a_{n}-a_{i}}\right) a_{n}-s\right)^{k-2}-(x-s)^{k-2} } \\
& \left.+\sum_{j=2}^{r+1}(-1)^{j}\left(\prod_{\substack{i=2 \\
i \neq k-j+1)}}^{k-1} \frac{x-a_{i}}{\mid a_{k-j+1}-a_{i}}\right) \frac{a_{n}-x}{a_{n}-a_{k-j+1}}\left(a_{k-j+1}-s\right)^{k-2}\right] .
\end{aligned}
$$

Repeating the above with $i=2, \ldots, k-r-1$ and $m=n$ or $k-j+1$ (once again $i \neq m$ always) on the last term each time, we finally obtain

$$
\begin{aligned}
& g_{k}^{r}(x, s)-(x-s)^{k-1}=\left(a_{n}-x\right) \sum_{l=0}^{k-r-2}(x-s)^{l}\left(\prod_{i=l+2}^{k-1}\left(x-a_{i}\right)\right)\left(s-a_{l+1}\right) \\
& \left\{\frac{\left(a_{n}-s\right)^{k-1-2}}{\prod_{i=1}^{k-1}\left(a_{n}-a_{i}\right)}+\sum_{i=2}^{r+1}(-1)^{j+1} \frac{\left(a_{k-j+1}-s\right)^{k-1-2}}{\prod_{i=l+1(i \neq k-j+1}^{k-1}\left|a_{k-j+1}-a_{i}\right|} \frac{1}{a_{n}-a_{k-j+1}}\right\} \\
& +(x-s)^{k-r-1}\left[\left(\prod_{i=k-r}^{k-1} \frac{x-a_{i}}{a_{n}-a_{i}}\right)\left(a_{n}-s\right)^{r}-(x-s)^{r}\right. \\
& \left.+\sum_{j=2}^{r+1}(-1)^{j}\left(\prod_{\substack{i=k-r \\
(i \neq k-j+1)}}^{k-1} \frac{x-a_{i}}{\left|a_{k-j+1}-a_{i}\right|}\right) \frac{a_{n}-x}{a_{n}-a_{k-j+1}}\left(a_{k-j+1}-s\right)^{r}\right] .
\end{aligned}
$$

Now (2.4) follows from the above in view of the fact that the factor multiplying $(x-s)^{k-r-1}$ is a polynomial of degree $r$ in $x$ and takes the value zero at $a_{k-r}, \ldots, a_{k-1}$ and $a_{n}$.

3. We first give some auxiliary results in the form of lemmas.

Lemma 3.1. For each $r(=1, \ldots, k-3)$ if $\rho$, a natural number, does not exceed $r$; then

$$
\frac{\left(a_{n}-s\right)^{\rho-1}}{\prod_{i=k-r}^{k-1}\left(a_{n}-a_{i}\right)}+\sum_{j=2}^{r+1}(-1)^{j+1} \frac{\left(a_{k-j+1}-s\right)^{\rho-1}}{\prod_{i=k-r(i \neq k-j+1}^{k-1}\left|a_{k-j+1}-a_{i}\right|} \frac{1}{a_{n}-a_{k-j+1}} \equiv 0
$$

and bence

$$
\left(\equiv A_{r, \rho}^{(k)}(s)\right)
$$

$$
\frac{\left(a_{n}-s\right)^{\rho}}{\prod_{i=k-r-1}^{k-1}\left(a_{n}-a_{i}\right)}+\sum_{j=2}^{r+1}(-1)^{j+1} \frac{\left(a_{k-j+1}-s\right)^{\rho}}{\prod_{i=k-r-1(i \neq k-j+1}^{k-1}\left|a_{k-j+1}-a_{i}\right|} \frac{1}{a_{n}-a_{k-j+1}}
$$

is divisible by $\left(a_{k-r-1}-s\right)^{\rho}$.

$$
\left(\equiv B_{r, \rho}^{(k)}(s)\right)
$$


Proof. That (3.2) has $\left(a_{k-r-1}-s\right)^{\rho}$ as a factor is immediate when relations (3.1) are known to be true as seen from the identity

$$
B_{r, \rho}^{(k)}(s)=\left(a_{k-r-1}-s\right)^{\rho} B_{r, 0}^{(k)}+\sum_{\sigma=0}^{\rho-1}\left(a_{k-r-1}-s\right)^{\sigma} A_{r, \rho-\sigma}^{(k)}(s)
$$

obtained by successive use of

$$
B_{r, \rho-\sigma}^{(k)}(s)=\left(a_{k-r-1}-s\right) B_{r, \rho-\sigma-1}^{(k)}+A_{r, \rho-\sigma}^{(k)}(s), \quad \sigma=0,1, \cdots, \rho-1 .
$$

To establish (3.1) for arbitrary $r(\leq k-3)$ and $\rho=1$, it is sufficient to observe that the polynomial

$$
1+\sum_{j=2}^{r+1}(-1)^{j+1}\left(\prod_{\substack{i=k-r \\ i \neq k-j+1)}}^{k-1} \frac{t-a_{i}}{\left|a_{k-j+1}-a_{i}\right|}\right)
$$

(of degree $r-1$ ) vanishes at $a_{k-r}, \ldots, a_{k-1}$ and hence identically. (This proves (3.1) when $r=1$.) Now assume that (3.1) holds when $r(>1)$ is replaced by $r-1$ and for $\rho=1, \ldots, \sigma(<r)$. Then, if $\rho=\sigma+1,(3.1)$ follows from the identity

$$
A_{r, \sigma+1}^{(k)}(s)=A_{r-1, \sigma}^{(k)}(s)+\left(a_{k-r}-s\right) A_{r, \sigma}^{(k)}(s), \quad 2 \leq r \leq k-3,1 \leq \sigma<r .
$$

This completes the proof by induction.

Remark. In the special case when $\rho=r,(3.2)$ is a polynomial of degree $r$. In view of the above result, we shall write this polynomial as $C_{r}^{(k)}\left(a_{k-r-1}-s\right)^{r}$, where $k$ is fixed and $C_{r}^{(k)}$ are constants for $r=1, \ldots, k-3$. In fact, $C_{r}^{(k)}=$ $B_{r, 0}^{(k)}$ as can be easily checked.

Lemma 3.2. For each $r(=1, \ldots, k-3), C_{r}^{(k)}\left(a_{k-r-1}-s\right)^{r}$ is positive on $\left(a_{k \rightarrow r-1}, a_{k-r}\right)$.

Proof. It is easy to check that for $r=1$.

$$
C_{1}^{(k)}\left(a_{k-2}-s\right) \equiv \frac{\left(s-a_{k-2}\right)}{\left(a_{n}-a_{k-2}\right)\left(a_{k-1}-a_{k-2}\right)}
$$

and thus, in addition to the conclusion, we have $C_{1}^{(k)}<0$.

Now assume that for $r=\sigma-1$, the conclusion is true and $\operatorname{sgn} C_{\sigma-1}^{(k)}=$ $(-1)^{\sigma-1}$. Then, if $r=\sigma$, we have 


$$
\begin{aligned}
& C_{\sigma}^{(k)}\left(a_{k-\sigma-1}-s\right)^{\sigma} \equiv\left(a_{k-\sigma-1}-s\right)^{\sigma-1} \\
& \cdot\left\{\frac{a_{n}-s}{\prod_{i=k-\sigma-1}^{k-1}\left(a_{n}-a_{i}\right)}+\sum_{j=2}^{\sigma+1}(-1)^{j+1} \frac{a_{k-j+1}-s}{\prod_{i=k-\sigma-1(i-k-j+1)}^{k-1}\left|a_{k-j+1}-a_{i}\right|} \frac{1}{a_{n}-a_{k-j+1}}\right\} \\
&\left(\equiv\left(a_{k-\sigma-1}-s\right)^{\sigma-1} H(s)\right),
\end{aligned}
$$

in view of

$$
B_{\sigma, \sigma}^{(k)}(s)=\left(a_{k-\sigma-1}-s\right)^{\sigma-1} B_{\sigma, 1}^{(k)}(s)
$$

Note that

$$
H\left(a_{k-\sigma-1}\right)=A_{\sigma, 1}^{(k)}\left(a_{k-\sigma-1}\right)=0 .
$$

Moreover, the sign of $H(s)$ is constant on $\left(a_{k-\sigma-1}, a_{k-\sigma}\right)$ and is that of $H\left(a_{k-\sigma}\right)$, namely

$$
\left(\prod_{\substack{i=k-\sigma-1 \\(i \neq k-\sigma)}}^{k-1}\left(a_{n}-a_{i}\right)\right)^{-1}+\sum_{j=2}^{\sigma}(-1)^{j+1}\left(\prod_{\substack{i=k-\sigma-1 \\ i \neq k-j+1, k-\sigma)}}^{k-1}\left|a_{k-j+1}-a_{i}\right|\right)^{-1}\left(a_{n}-a_{k-j+1}\right)^{-1}
$$

which is $\operatorname{sgn} q\left(a_{n}\right)$, where

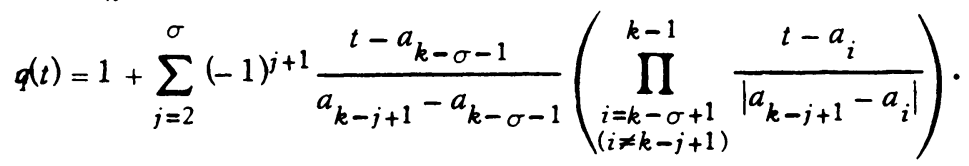

Also, sgn $C_{\sigma-1}^{(k)}=\operatorname{sgn} p\left(a_{n}\right)$, where

$$
p(t)=1+\sum_{j=2}^{\sigma}(-1)^{j+1} \prod_{\substack{i=k-\sigma \\ i \neq k-j+1)}}^{k-1} \frac{t-a_{i}}{\left|a_{k-j+1}-a_{i}\right|} .
$$

In view of the facts that both polynomials $p(t)$ and $q(t)$ are of degree $\sigma-1$, have the same zeros $a_{k-\sigma+l}(l=1,2, \ldots, \sigma-1)$, and $p\left(a_{k-\sigma}\right)=q\left(a_{k-\sigma-1}\right)=1$, it follows that $\operatorname{sgn} H\left(a_{k-\sigma}\right)=\operatorname{sgn} C_{\sigma-1}^{(k)}$. Thus, $C_{\sigma}^{(k)}\left(a_{k-\sigma-1}-s\right)^{\sigma}$ is positive in $\left(a_{k-\sigma-1}, a_{k-\sigma}\right)$ and $\operatorname{sgn} C_{\sigma}^{(k)}=(-1)^{\sigma}$. This completes the proof.

Lemma 3.3. For all integers $k, r, m$ such that $4 \leq k(<n), 1 \leq r \leq k-3$, and $r \leq m \leq k-2$,

$$
\begin{aligned}
A(k, r, m, s) \equiv & \frac{\left(a_{n}-s\right)^{m}}{\prod_{i=k-m-1}^{k-1}\left(a_{n}-a_{i}\right)} \\
& +\sum_{i=2}^{r+1}(-1)^{j+1} \frac{\left(a_{k-j+1}-s\right)^{m}}{\prod_{i=k-m-1(i \neq k-j+1}^{k-1}\left|a_{k-j+1}-a_{i}\right|} \frac{1}{a_{n}-a_{k-j+1}}
\end{aligned}
$$

is nonnegative on $\left[a_{k-r-1}, a_{k-r}\right]$. 
Proof. First observe that the assertion follows from Lemma 3.2 if $m=r$ and $k$ arbitrary, admissible. Also, if $r=1$ and $m, k$ admissible, then the identity

$$
\begin{gathered}
\left(\frac{a_{n}-s}{a_{n}-a_{k-m-2}}-\frac{a_{k-1}-s}{a_{k-1}-a_{k-m-2}}\right) \frac{\left(a_{n}-s\right)^{m}}{\prod_{i=k-m-1}^{k-1}\left(a_{n}-a_{i}\right)} \\
\equiv \frac{\left(a_{n}-s\right)^{m}}{\prod_{i=k-m-2}^{k-2}\left(a_{n}-a_{i}\right)} \frac{s-a_{k-m-2}}{a_{k-1}-a_{k-m-2}},
\end{gathered}
$$

in view of $a_{k-1} \geq s \geq a_{k-2}>a_{k-m-2}$, implies

$$
\frac{\left(a_{n}-s\right)^{m+1}}{\prod_{i=k-m-2}^{k-1}\left(a_{n}-a_{i}\right)} \geq \frac{a_{k-1}-s}{a_{k-1}-a_{k-m-2}} \frac{\left(a_{n}-s\right)^{m}}{\prod_{i=k-m-1}^{k-1}\left(a_{n}-a_{i}\right)} .
$$

Thus $A(k, 1, m, s)$ is nonnegative by using induction on $m$.

Now we may assume $r \geq 2$ and thus admissible $k \geq 5$. Let the conclusion be true about $A(k, r, m, s)$ for admissible $r, k$. Note that in addition to (3.4) we have the identities

$$
\left(\frac{a_{k-1}-s}{a_{k-1}-a_{k-m-2}}-\frac{a_{k-j+1}-s}{a_{k-j+1}-a_{k-m-2}}\right) \frac{\left(a_{k-j+1}-s\right)^{m}}{\prod_{i=k-m-1(i \neq k-j+1}^{k-1}\left|a_{k-j+1}-a_{i}\right|}
$$

$$
\begin{array}{r}
\equiv \frac{\left(a_{k-j+1}-s\right)^{m}}{\prod_{i=k-m-2(i \neq k-j+1}^{k-2}\left|a_{k-j+1}-a_{i}\right|} \frac{s-a_{k-m-2}}{a_{k-1}-a_{k-m-2}}, \\
j=3, \cdots, r+1 .
\end{array}
$$

Multiplying each $\left(3.5_{j}\right)$ by $(-1)^{j}\left(a_{n}-a_{k-j+1}\right)^{-1}$ and adding all to (3.4) we get

$$
\begin{gathered}
A(k, r, m+1, s)=\frac{a_{k-1}-s}{a_{k-1}-a_{k-m-2}} A(k, r, m, s)+\frac{s-a_{k-m-2}}{a_{k-1}-a_{k-m-2}} B, \\
B=A(k-1, r-1, m, s) .
\end{gathered}
$$

By induction hypothesis $A(k, r, m, s)$ as well as $A(k-1, r-1, m, s)$ are nonnegative on $\left[a_{k-r-1}, a_{k-r}\right]$ in view of the admissibility of $k-1$ and $r-1$ in addition to that of $k$ and $r$.

The following theorem is the main result which leads to (1.3).

Theorem 3.4. For $g_{k}(x, s)$ the following bolds: 


$$
\operatorname{sgn} g_{k}(x, s)= \begin{cases}1, & (x, s) \in\left[a_{k-1}, a_{n}\right] \times\left[a_{1}, a_{n}\right], \\ (-1 Y, & (x, s) \in\left[a_{k-r-1}, a_{k-r}\right] \times\left[a_{1}, a_{n}\right], \quad r=1, \ldots, k-2 .\end{cases}
$$

Proof. First we consider the triangle $a_{1} \leq s \leq x \leq a_{n}$. The conclusion about sgn $g_{k}(x, s)$ in this triangle is obvious from (2.2) when $s \leq a_{2}$, and immediate when $a_{k-1} \leq s \leq a_{n}$ since $\left(x-a_{i}\right)\left(a_{n}-s\right) \geq\left(a_{n}-a_{i}\right)(x-s)$ for $i=1, \ldots, k-1$. Also, if for $r=1, \ldots, k-3, s \in\left[a_{k-r-1}, a_{k-r}\right]$, then the assertion about sgn $g_{k}(x, s)$ follows from (2.4) in view of Lemma 3.3, noting that $l+1=k-m-1$ and that

$$
\operatorname{sgn}\left(\prod_{i=k-m}^{k-1}\left(x-a_{i}\right)\right)= \begin{cases}1, & x \in\left[a_{k-1}, a_{n}\right], \\ (-1)^{r}, & x \in\left[a_{k-r-1}, a_{k-r}\right] .\end{cases}
$$

To discuss the triangle $a_{1} \leq x \leq s \leq a_{n}$, we begin by observing that if $s \geq$ $a_{k-1}$, then (2.2) at once gives the conclusion. For $s \leq a_{k-1}$, we use induction. First note that $g_{3}(x, s)$ has the asserted signs. Now assume that $g_{m}(x, s)$ has the asserted signs. Then (2.3) shows that if $x \in\left[a_{m-1}, a_{m}\right],-\operatorname{sgn} g_{m+1}(x, s) \geq 0$. Also, noting that if $x \in\left[a_{m-r}, a_{m-r+1}\right]$ where $r=2, \ldots, m-1$, then $\operatorname{sgn} g_{m}(x, s)$ $=\operatorname{sgn}\left(\Pi_{i=1}^{m-1}\left(x-a_{i}\right)\right)$, we have the desired conclusion for $k=m+1$.

This completes the proof.

Theorem 3.5. For any $k(\leq n)$ the following bolds:

$$
\int_{a_{1}}^{a_{n}}\left|g_{k}(x, s)\right| d s=\frac{1}{k !}\left(x-a_{1}\right)\left(a_{n}-x\right)\left(\prod_{i=2}^{k-1}\left|x-a_{i}\right|\right) .
$$

Proof. In view of Theorem 3.4,

$$
\int_{a_{1}}^{a}\left|g_{k}(x, s)\right| d s=\left|\int_{a_{1}}^{a} g_{k}(x, s) d s\right| .
$$

The value of the integral on the right-hand side by $(2.2)$ is

$$
\begin{aligned}
& \frac{1}{k !}\left[\left(\prod_{i=2}^{k-1} \frac{x-a_{i}}{a_{i}-a_{1}}\right) \frac{a_{n}-x}{a_{n}-a_{1}}\left(a_{2}-a_{1}\right)^{k}+\left(\prod_{i=1}^{k-1} \frac{x-a_{i}}{a_{n}-a_{i}}\right)\left(a_{n}-a_{2}\right)^{k}+(-1)^{k-1}\left(a_{2}-x\right)^{k}\right] \\
& +\frac{1}{(k-1) !} \sum_{r=1}^{k-3} \int_{a_{k-r-1}}^{a_{k-r}}\left\{\sum_{j=2}^{r+1}(-1)^{j}\left(\prod_{\substack{i=1 \\
(i \neq k-j+1)}}^{k-1} \frac{x-a_{i}}{\left|a_{k-j+1}-a_{i}\right|}\right) \frac{a_{n}-x}{a_{n}-a_{k-j+1}}\left(a_{k-j+1}-s\right)^{k-1}\right\} d s, \\
& \frac{1}{k !}\left[\left(\prod_{i=2}^{k-1} \frac{x-a_{i}}{a_{i}-a_{1}}\right) \frac{\left.a_{n}-a_{2}\right]}{a_{n}-a_{1}}\left(a_{2}-a_{1}\right)^{k}+\left(\prod_{i=1}^{k-1} \frac{x-a_{i}}{a_{n}-a_{i}}\right)\left(a_{n}-a_{2}\right)^{k}-\left(x-a_{2}\right)^{k}\right] \quad \\
& +\frac{1}{(k-1) !} \sum_{r=1}^{k-3} \int_{a_{k-r-1}}^{a_{k-r}}\left\{\sum_{j=2}^{r+1}(-1)^{j}\left(\prod_{\substack{i=1 \\
(i \neq k-1}}^{k} \frac{x-a_{i}}{\left|a_{k-j+1}-a_{i}\right|}\right) \frac{a_{n}-x}{a_{n}-a_{k-j+1}}\left(a_{k-j+1}-s\right)^{k-1}\right\} d s, \\
& x \in\left[a_{k-1}, a_{n}\right] \text { or } x \in\left[a_{k-1-1}, a_{k-1}\right], l=1, \ldots, k-3 .
\end{aligned}
$$


Thus, whatever $x \in\left[a_{1}, a_{n}\right]$,

$\int_{a_{1}}^{a} g_{k}(x, s) d s$

$$
\begin{aligned}
=\frac{1}{k !}\left[\left(\prod_{i=2}^{k-1} \frac{x-a_{i}}{a_{i}-a_{1}}\right) \frac{a_{n}-x}{a_{n}-a_{1}}\left(a_{2}-a_{1}\right)^{k}+\left(\prod_{i=2}^{k-1} \frac{x-a_{i}}{a_{n}-a_{i}}\right)\left(a_{n}-a_{2}\right)^{k}-\left(x-a_{2}\right)^{k}\right. \\
\left.+\sum_{j=2}^{k-2}(-1)^{j}\left(\prod_{\substack{i=1 \\
i \neq k-j+1)}}^{k-1} \frac{x-a_{i}}{\left|a_{k-j+1}-a_{i}\right|}\right) \frac{a_{n}-x}{a_{n}-a_{k-i+1}}\left(a_{k-j+1}-a_{2}\right)^{k}\right] .
\end{aligned}
$$

It is easily seen that the expression in (3.8) is a polynomial (in $x$ ) of ciegree $k$ which has zeros $a_{1}, \ldots, a_{k-1}$ and $a_{n}$. Moreover, the coefficient of $x^{k}$ is $-1 / k$, hence the conclusion in (3.6).

4. Applications. In this section $k=n$. Thus, consider the ordinary differential equation

$$
y^{(n)}+f\left(x, y, y^{\prime}, \cdots, y^{(n-1)}\right)=0
$$

where $f$ is continuous on $\left[a_{1}, a_{n}\right] \times R^{n}$ and satisfies

$$
\left|f\left(x, y, y^{\prime}, \ldots, y^{(n-1)}\right)\right| \leq K|y|
$$

(The above hypothesis is evidently no more restrictive than that of Beesack-see (3.2) in [1].)

The following lemma gives a bound which is better than (2.13) of [1] in situations which are not "highly pathological" (see Remark below).

Lemma 4.1. Let $x \in\left[a_{1}, a_{n}\right]$. Then,

$$
\prod_{i=1}^{n}\left|x-a_{i}\right| \leq(n-1)^{n-1}\left(\frac{\delta}{2}\right)^{n},
$$

where $a_{1} \leq a_{2} \leq \cdots \leq a_{n}, \delta=\max _{2 \leq i \leq n}\left(a_{i}-\dot{a}_{i-1}\right)$.

Proof. Let $x \in\left(a_{r}, a_{r+1}\right)$, where $r(\geq 1)<[(n+1) / 2]$, the integral part of $(n+1) / 2$. Then, 


$$
\begin{aligned}
{\left[\left\{(n-2 r+1)\left(x-a_{1}\right)\right\}\left(x-a_{2}\right) \cdots\left(x-a_{r}\right) \prod_{i=r+1}^{n}\left(a_{i}-x\right)\right]^{1 / n} } \\
\quad \leq \frac{1}{n}\left(\sum_{i=r+1}^{n}\left(a_{i}-a_{1}\right)-\sum_{i=1}^{r}\left(a_{i}-a_{1}\right)\right) \\
\quad=\frac{1}{n}\left(\sum_{i=1}^{r-1}(n-2 r+i)\left(a_{i+1}-a_{i}\right)+\sum_{i=r}^{n-1}(n-i)\left(a_{i+1}-a_{i}\right)\right) \\
\leq \frac{n^{2}-n-2 r(r-1)}{2 n} \delta,
\end{aligned}
$$

that is

$$
\prod_{i=1}^{n}\left|x-a_{i}\right| \leq \frac{1}{n-2 r+1}\left(\frac{\delta}{2 n}\right)^{n}\left(n^{2}-n-2 r(r-1)\right)^{n} .
$$

Similarly, if $[(n+1) / 2] \leq r<n$, we have

$$
\prod_{i=1}^{n}\left|x-a_{i}\right| \leq \frac{1}{n-2(n-r)+1}\left(\frac{\delta}{2 n}\right)^{n}\{n(n-1)-2(n-r)(n-r-1)\}^{n} .
$$

It is easy to check that

$$
f(r)=\{n(n-1)-2 r(r-1)\}^{n} /(n-2 r+1)
$$

is nonincreasing for $(1 \leq) r<[(n+1) / 2]$ and $f(n-r)$ is nondecreasing for $([(n+1) / 2] \leq) r<n$. The estimate (4.3) follows in view of $f(1)=f(n-1)=$ $n^{n}(n-1)^{n-1}$.

Remark. The bound in (4.3) is better than Beesack's if and only if

$$
\delta<2\left(a_{n}-a_{1}\right) / n \text {. }
$$

If $n \geq 3$, this is always the case when the $a_{i}$ 's are equally spaced. In general, however, (2.13) of [1] gives a sort of best possible bound.

Theorem 4.2. Let the boundary value problem (4.1) and

$$
y\left(a_{1}\right)=\cdots=y\left(a_{n}\right)=0, \quad a_{1}<a_{2}<\cdots<a_{n},
$$

bave a solution. Then,

$$
K^{-1}< \begin{cases}\frac{(n-1)^{n-1}}{n !}\left(\frac{\delta}{2}\right)^{n}, & \text { if } \delta<\frac{2}{n}\left(a_{n}-a_{1}\right), \\ \frac{(n-1)^{n-1}}{n^{n}} \frac{\left(a_{n}-a_{1}\right)^{n}}{n !}, & \text { otherwise, }\end{cases}
$$

where $K$ and $\delta$ are as above. 
Proof. (4.5) follows from the fact that $y(x)$ satisfies the integral equation

$$
y(x)=\int_{a_{1}}^{a_{n}} g_{n}(x, s) f\left(s, y(s), \ldots, y^{(n-1)}(s)\right) d s, \quad x \in\left[a_{1}, a_{n}\right],
$$

and thus identifying $x$ with a point where $|y(x)|$ attains its maximum, we have

$$
1<K \int_{a_{1}}^{a}\left|g_{n}(x, s)\right| d s,
$$

in view of (4.2).

Remark. The above result is an improvement on Beesack's necessary condition whenever the function $b(x)$ in his (3.2) is constant (of course, multiple zeros are not allowed). Apart from the case $b(x) \equiv K$, the two results are not comparable.

Next turning to the question of obtaining a lower bound for the $m$ th zero of solutions of the linear differential equation

$$
y^{(n)}+p(x) y=0
$$

we state the following result:

Theorem 4.3. Let $p(x)$ in (4.8) be continuous and bounded on $[a, \infty)$. If $a_{1}(\geq a)<a_{2}<\cdots<a_{m}$ are consecutive simple zeros of a solution of (4.8), then for $m>n$

$$
a_{m}>a_{1}+\left(\frac{(m-n+1) n !}{K}\left(\frac{n}{n-1}\right)^{n-1}\right)^{1 / n},
$$

where $|p(x)| \leq K$.

We omit the proof which is a straightforward adaptation of the above proof and of the proof of (3.15) in [1].

Remark. As in [1], if $m>2 n-1$, in place of (4.9) we have the estimate

$$
a_{m}>a_{1}+(n /(n-1))((m-n) n ! / K)^{1 / n} \text {. }
$$

Acknowledgement. The authors thank the referee for helpful suggestions.

\section{REFERENCES}

1. P. R. Beesack, On the Green's function of an N-point boundary value problem, Pacific J. Math. 12 (1962), 801-812. MR 26 \#2672.

2. Z. Nehari, On an inequality of P. R. Beesack, Pacific J. Math. 14 (1964), 261263. MR $28 \# 3192$.

3. D. V. V. Wend, On the zeros of solutions of some linear complex differential equations, Pacific J. Math. 10 (1960), 713-722. MR 22 \#9657.

DEPARTMENT OF MATHEMATICS, INDIAN INSTITUTE OF TECHNOLOGY, MADRAS 600036 , 